\title{
BINARY GAS MIXTURE ANALYSIS USING A TWO-DIMENSIONAL ARRAY OF INTERDIGITATED GATE ELECTRODE FIELD-EFFECT TRANSISTORS
}

\author{
John M. Wiseman and Edward S. Kolesar
}

\section{Texas Christian University, Department of Electrical Engineering}

Fort Worth, TX 76129-0001

\section{ABSTRACT}

Single component and binary gas mixtures composed of nitrogen dioxide $\left(\mathrm{NO}_{2}\right)$ and ammonia $\left(\mathrm{NH}_{3}\right)$ were analyzed with a microsensor composed of an array of Interdigitated Gate Electrode Field Effect Transistor (IGEFET) sensor elements coated with copper-, nickel-, and cobalt-phthalocyanine thin films. The design and mode of operation of the IGEFET microsensor facilitated the near-simultaneous measurement of the direct current (DC) and alternating current (AC) electrical response of the metal-substituted phthalocyanine (MPc) thin films upon exposure to the challenge gas mixtures. To facilitate predicting the challenge gas component concentrations for mixtures containing 100-4000 parts-per-billion (ppb) $\mathrm{NO}_{2}$ and 20-400 parts-per-million (ppm) $\mathrm{NH}_{3}$, the principal component analysis (PCA) method and multilinear regression technique were applied to the distinctive features identified in the measured response of the IGE structure's DC resistance and the IGEFET transfer function's gain and phase.

\section{INTRODUCTION}

The detection and measurement of chemical species, as well as the control of chemical reactions, have widespread applications. Of particular interest with respect to the environment, is the need for small and autonomously operating microsensors that can accomplish the toxic gas monitoring function. Two important classes of environmentally-sensitive compounds include the oxides of nitrogen, especially nitrogen dioxide $\left(\mathrm{NO}_{2}\right)$, and ammonia $\left(\mathrm{NH}_{3}\right)$.

One type of chemical microsensor technology is the Interdigitated Gate Electrode Field Effect Transistor (IGEFET). As depicted in Figure 1, the IGEFET can be partitioned into two fundamental components: the integrated circuit (IC) and the chemically-sensitive thin film deposited on the surface of the interdigitated gate electrode (IGE) structure of each IGEFET sensing element.

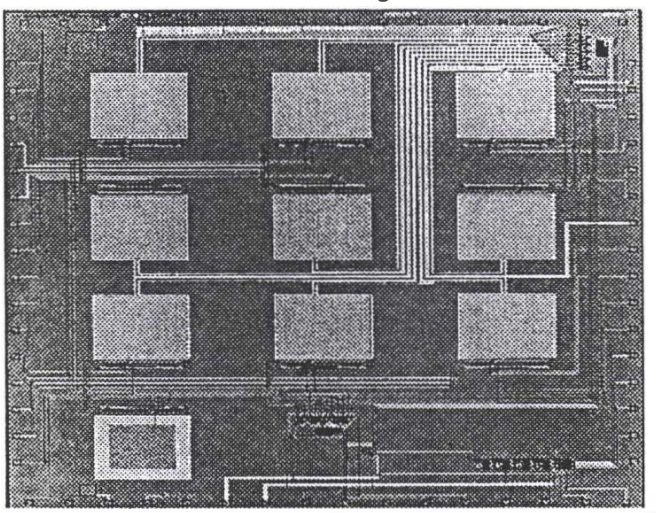

(a)

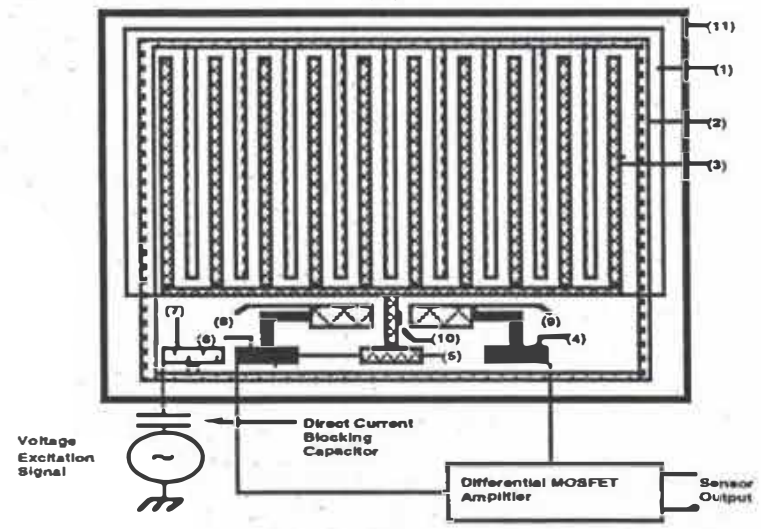

(b)

Figure 1. (a) IGEFET microsensor IC depicting a $3 \times 3$ two-dimensional array of identical sensors whose independent set of responses can be electrically-multiplexed with the in situ circuitry. (The overall dimensions of the IC are $4466 \times 6755 \mu \mathrm{m}$. Each IGE structure con- sists of 34 foating-electrode fingers and 35 driven-electrode fingers. Each finger is $10 \mu \mathrm{m}$ wide, and the inter-electrode finger spacing is 10 $\mu \mathrm{m}$. The chemically-active area of the IGE measures $1370 \times 1370$ $\mu \mathrm{m}$.$) (b) IGEFET physical structure and electrical connections.$ Legend: (I) copper phthalocyanine (CuPc) thin film, (2) drivenelectrode, (3) floating-electrode, (4) MOSFET drain contact, (5) MOSFET gate and floating-electrode contact, (6) MOSFET source contact, (7) driven-electrode contact, (8) MOSFET source, (9) MOSFET drain, (10) MOSFET drain-to-source channel, and (II) host silicon substrate.

\section{EXPERIMENTAL}

To facilitate predicting the challenge gas component concentrations for mixtures containing 100-4000 parts-per-billion (ppb) $\mathrm{NO}_{2}$ and 20-400 parts-per-million (ppm) $\mathrm{NH}_{3}$, the principal component analysis (PCA) method and multilinear regression [1] technique were applied to the distinctive features identified in the measured response of the IGE structure's DC resistance and the IGEFET transfer function's gain and phase. As depicted in Figure $2(a)-(c)$, baseline drift of the IGEFET microsensor was minimized by normalizing the extracted features. The individual challenge gas components $\mathrm{NO}_{2}$ and $\mathrm{NH}_{3}$ ) were discriminated on the basis of the opposite changes (sign change) they induced in the MPc-coated IGEFET microsensor's electrical properties, the different gas sensitivities revealed by the MPc-films, the time dependence of the electrical changes induced by a challenge gas exposure, and finally, the different gas sensitivities of the frequency-dependent components manifested within the MPccoated IGEFET transfer function. As depicted in Figure $2(d)-(f)$, the predicted challenge gas component concentrations were always within $50 \%$ of the known concentrations for all of the gas mixtures analyzed. As anticipated, the smallest error [ $26 \%$ for $\mathrm{NO}_{2}$, Figure 2 (e) and $14 \%$ for $\mathrm{NH}_{3}$, Figure $\left.2(f)\right]$ was achieved with the single component analysis of each challenge gas using the normalized IGE structure's DC resistance response. The error in the predicted single component analysis of each challenge gas was slightly larger when the analysis was accomplished with the IGEFET transfer function's gain and phase. For the binary gas mixture analysis, the smallest error in the predicted challenge gas concentrations (approximately $25 \%$ for each component) was achieved with the IGEFET transfer function's gain response. The phase response was the least effective information for predicting the concentrations of the challenge gas components. As a consequence of the favorable results obtained from the IGEFET transfer function's gain response, a complementary approach was investigated. This technique utilized a lumped-element equivalent circuit model that accurately predicts the vanations observed in the IGEFET transfer function's gain and phase Bode plots. When the $\mathrm{NO}_{2}$ and $\mathrm{NH}_{3}$ analyses were performed with the conductance parameters extracted from the lumped-element equivalentcircuit model [2], the predicted gas concentration errors were significantly less than the corresponding errors obtained with the gain and phase response feature analyses, and it was never larger than the error predicted when the normalized DC resistance response was used.
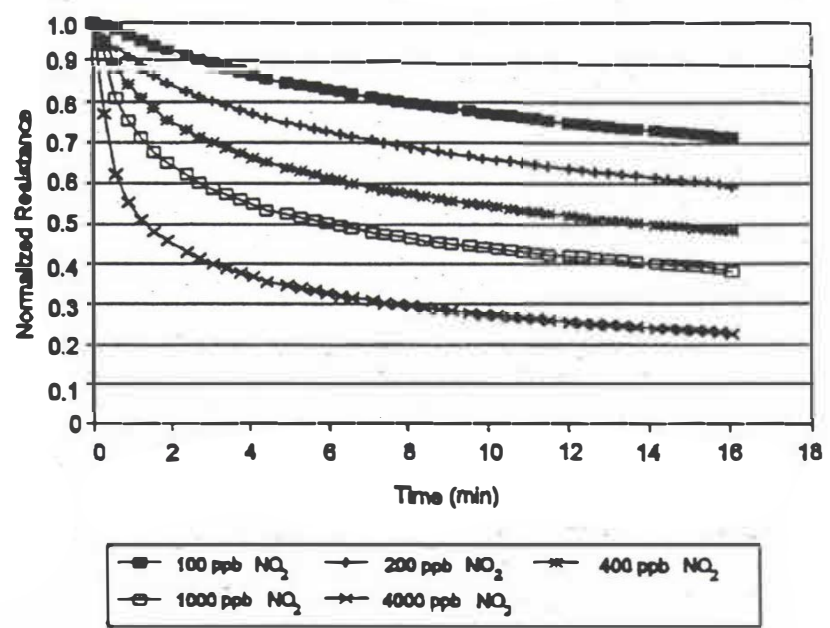

(a) 

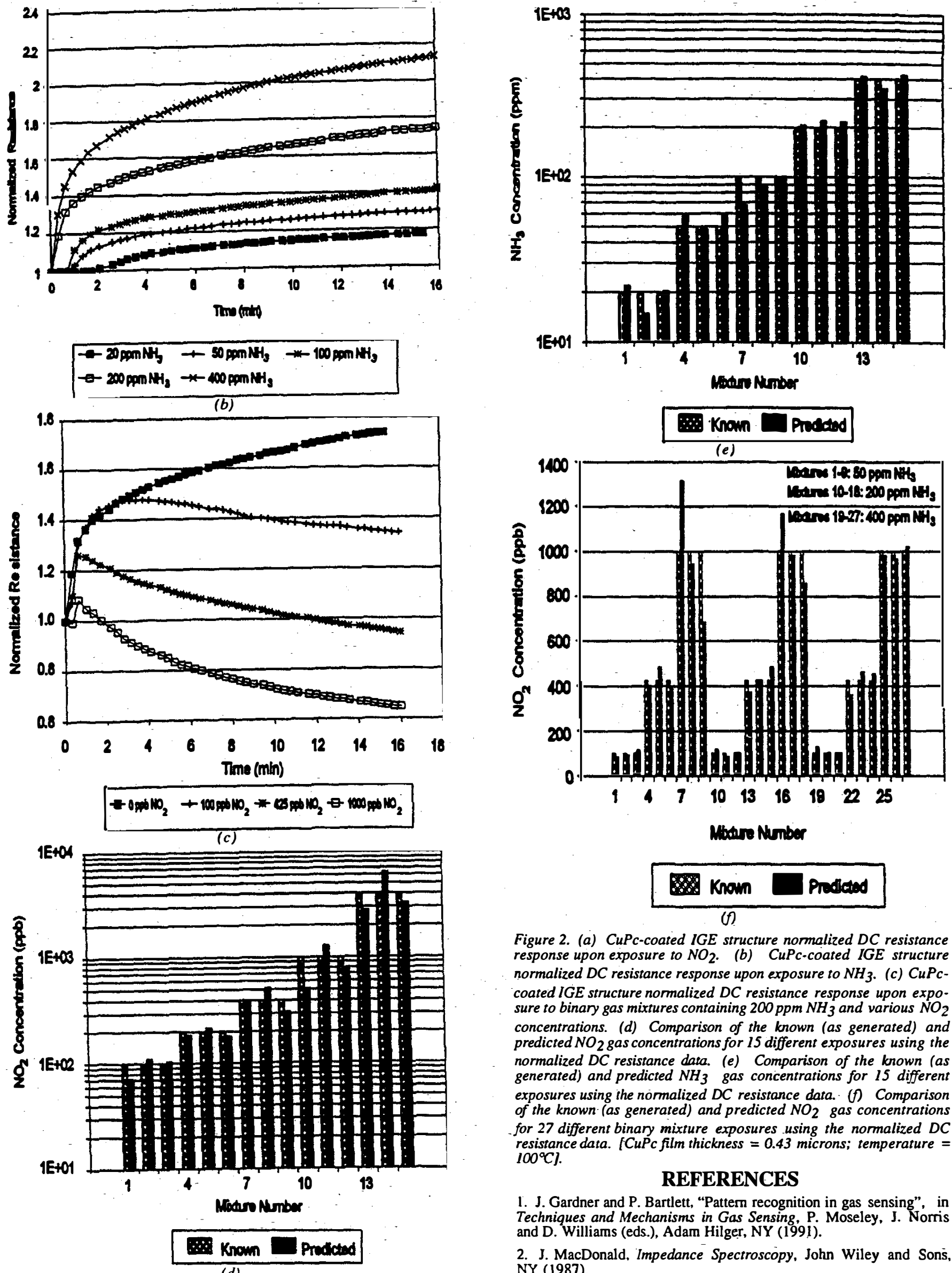

Figure 2. (a) CuPc-coated IGE structure normalized DC resistance response upon exposure to $\mathrm{NO}_{2}$. (b) CuPc-coated IGE structure normalized DC resistance response upon exposure to $\mathrm{NH}_{3}$. (c) $\mathrm{CuPc}$ coated IGE structure normalized $D C$ resistance response upon exposure to binary gas mixtures containing 200 ppm $\mathrm{NH}_{3}$ and various $\mathrm{NO}_{2}$ concentrations. (d) Comparison of the known (as generated) and predicted $\mathrm{NO}_{2}$ gas concentrations for 15 different exposures using the normalized DC resistance data. (e) Comparison of the known (as generated) and predicted $\mathrm{NH}_{3}$ gas concentrations for 15 different exposures using the normalized DC resistance data. (f) Comparison of the known (as generated) and predicted $\mathrm{NO}_{2}$ gas concentrations for 27 different binary mixture exposures using the normalized DC resistance data. $[\mathrm{CuPc}$ film thickness $=0.43$ microns; temperature $=$ $100^{\circ} \mathrm{C}$.

\section{REFERENCES}

I. J. Gardner and P. Bartlett, "Pattem recognition in gas sensing", in Techniques and Mechanisms in Gas Sensing, P. Moseley, J. Norris and D. Williams (eds.), Adam Hilger, NY (1991).

2. J. MacDonald, Impedance Spectroscopy, John Wiley and Sons, NY (1987). 